\title{
Random forest model for feature-based Alzheimer's disease conversion prediction from early mild cognitive impairment subjects
}

\author{
Matthew Velazquez ${ }^{1^{*}}$, Yugyung Lee ${ }^{1}$, \\ for the Alzheimer's Disease Neuroimaging Initiative \\ 1 Department of Computer Science, University of Missouri - Kansas City, Kansas City, \\ MO, USA
}

9Membership of the Alzheimer's Disease Neuroimaging Initiative can be found in the Acknowledgments section.

*mv3md@mail.umkc.edu

\begin{abstract}
Alzheimer's Disease (AD) conversion prediction from the mild cognitive impairment (MCI) stage has been a difficult challenge. This study focuses on providing an individualized MCI to $\mathrm{AD}$ conversion prediction using a balanced random forest model that leverages clinical data. In order to do this, 383 Early Mild Cognitive Impairment (EMCI) patients were gathered from the Alzheimer's Disease Neuroimaging Initiative (ADNI). Of these patients, 49 would eventually convert to AD (EMCI_C), whereas the remaining 335 did not convert (EMCI_NC). All of these patients were split into training and testing data sets with 95 patients reserved for testing. Nine clinical features were selected, comprised of a mix of demographic, brain volume, and cognitive testing variables. Oversampling was then performed in order to balance the initially imbalanced classes. Our results showed that a random forest model was effective (93.6\% accuracy) at predicting the conversion of EMCI patients to AD based on these clinical features. Additionally, we assessed the importance of each clinical feature at both the individual and model level for interpretation of the prediction itself. Our model could impact the clinical environment as a tool to predict the conversion to $\mathrm{AD}$ from a prodromal stage or to identify ideal candidates for clinical trials.
\end{abstract}

\section{Introduction}

Alzheimer's Disease (AD) is a progressive, degenerative brain disorder that leads to nerve cell death and tissue loss in the brain. Currently, there are no treatment plans that prevent the progression of $\mathrm{AD}$, and this has led to increased emphasis on being able to predict $\mathrm{AD}$ at an earlier stage. Mild Cognitive Impairment (MCI) is an intermediary stage between being cognitively normal and having $\mathrm{AD}$ where $32 \%$ of MCI patients will go on to develop Alzheimer's Disease [1]. This makes the MCI stage an ideal target for early prediction as studies point to early diagnosis as being key to potentially delaying the overall progression of $\mathrm{AD}$ [1]. Early detection at the MCI stage can assist in clinical trial enrollment and provide more specific treatment plans when more effective ones do become available. Our focus in this study was to target the earliest subset of MCI patients (EMCI), as that subset is the furthest from an $\mathrm{AD}$ diagnosis and would thus provide a more beneficial prediction. As a result, it is of high importance to accurately determine which EMCI patients will develop AD. 
For this reason, an accurate, ensemble learning model that can aid in clinical decision making is necessary to help ascertain the patient's prognosis. Random Forest is a supervised learning algorithm that randomly creates and merges multiple decision trees and has been proficient with classification problems [2]. In our work, this Random Forest is used to determine which patients will convert to AD (our EMCI_C class) and those that will not convert (EMCI_NC) against an imbalanced data set. As well as determining how to best balance the data, assessing which clinical features are most relevant for conversion prediction is fundamental to our problem. Through Random Forest we are able to see which of our clinical features has the most significant impact at both the model and the individual prediction levels. This allows us to interpret the individual results better to provide more clinical significance. In this study, we sought to (1) identify significant features from clinical data; (2) build a random forest classification model from an imbalanced data set of those features; (3) determine the prediction accuracy of our model.

Also, we observed the associations between individual predictors and their importance to the problem. By attempting different feature groupings, we were able to distinguish the most crucial feature types. As a result of this approach, our work provides a clinical decision-making tool that can predict MCI-to-AD conversion with high accuracy and interpret the results meaningfully. We envision that this work will provide an accurate tool for predicting conversion probability from MCI to AD and further understand the impact of neuropsychological, biomarker, and demographic features.

\section{Related work}

A review on the use of random forest models in classifying Alzheimer's Disease was provided by Sarica et al. [3]. Their review consisted of 12 studies that were primarily focused on the classification of Alzheimer's Disease stages from MRI images. The accuracy across these studies ranged from $53 \%$ to $96 \%$, depending on whether they were performing multiclass classification or not. These studies were also focused on the direct stage classification of AD vs. Normal Controls vs. MCI, rather than the prediction of AD from an earlier stage.

Another review by Weiner et al. [4] summarized 49 ADNI papers. These papers did target the prediction of AD but were also focused mainly on MRI data. These were occasionally supplemented by clinical data or other imaging data (PET), with most studies using a support vector machine (SVM) model. A few of the studies [5] [6] [7] did use the random forest algorithm as well and will be compared, alongside the SVM implementations, against our model's performance.

Huang et al. [8] proposed a predictive nomogram that combined image features, clinical factors, and $\mathrm{AB}$ concentration to predict the conversion of MCI to AD. They also explored the associations between the different selected features and reported on their significance. Their goal was to examine the associations at both a macro and micro level to better understand the underlying patterns.

Moore et al. [9] proposed using a pairwise selection from time-series data to predict AD conversion. The authors analyzed the relationships between data point pairs at different times using a random forest algorithm. They leveraged a mix of demographic and genetic data and achieved a classification accuracy of $73 \%$ as a result.

Lebedev et al. [6] used a combination of structural MRI scans along with a few clinical features from the ADNI data set to achieve an MCI-to-AD conversion accuracy of $81.3 \%$. Their work also saw a sharp increase in accuracy by using a Random Forest algorithm rather than a Support Vector Machine. One advantage in their study is that they validated the model extensively outside of the ADNI data set and found no 
Table 1. EMCI Data set for Machine Learning

\begin{tabular}{l|c|c}
\hline & EMCI_C & EMCI_NC \\
\hline Subject\# & 49 & 335 \\
\hline Visit\# & 198 & 1608 \\
\hline Record\# after Over-sampling & 1608 & 1608 \\
\hline Training Data & 1206 & 1206 \\
\hline Testing Data & 402 & 402 \\
\hline
\end{tabular}

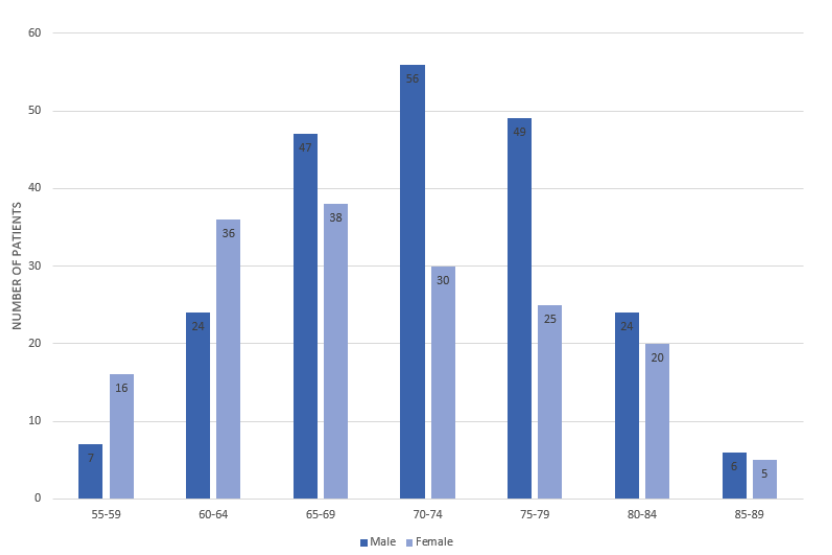

Fig 1. Participants' Age and Gender Distribution

substantial drop in accuracy, suggesting a good foundation for clinical implementation.

\section{Methods}

\section{Alzheimer's disease neuroimaging initiative data}

All data used for this paper were obtained from the Alzheimer's Disease Neuroimaging Initiative (ADNI) database and included patients from their ADNI-1, ADNI-2, and ADNI-GO studies [10]. "The ADNI was launched in 2003 as a public-private

partnership with the primary goal of testing whether serial magnetic resonance imaging (MRI), positron emission tomography (PET), other biological markers, and clinical and neuropsychological assessment can be combined to measure the progression of mild cognitive impairment (MCI) and early Alzheimer's disease (AD)" [10]. Early Mild Cognitive Impairment (EMCI) patients were eligible for our study as long as they had follow-up appointments for greater than a year. The EMCI subset consists of patients that are 5-7 years before a possible AD diagnosis and are identified by the results of the Wechsler Memory Scale Logical Memory II test. These EMCI patients were then subdivided into two groups based on whether they would eventually be diagnosed with Alzheimer's Disease or not. We chose to represent these groups as EMCI_C, for our AD conversion group, and EMCI_NC for our stable group. From the ADNI variables, the Clinical Dementia Rating was used to make this determination based on the value of their last visit's diagnosis. The remaining 1806 EMCI visits were then used as a starting point for training prior to augmentation. Of these, 198 belonged to the EMCI_C class while 1608 visits were from EMCI_NC subjects. Overall, our study consisted of 383 EMCI patients (shown in Figure 1), with 49 belonging to the EMCI_C group and the remaining 335 within the EMCI_NC group. $75 \%$ (288 patients) of our selected patients were used to train the random forest model, with the remaining 25\% (95 patients) used for validation testing. 


\section{Clinical features selection}

The clinical features that were used to train our random forest model included a mix of genetic biomarkers (APOE4), physical biomarkers (hippocampal and ventricular volume), four neuropsychological scale scores (ADAS13, ADAS11, FAQ, MMSE), and the patient's demographic information (age, race). Many different variations of ADNI features were tested for model inclusion; however, these nine features were found to provide the best overall fit. Additionally, related studies have used similar features and found the mix of biomarker and neuropsychological scale scores to be an ideal selection for AD prediction [11]. The ADNI features that we have used per training group can be seen in Table 2.

Table 2. ADNI Clinical Features and EMCI Patient Characteristics Used for Random Forest Model Training

\begin{tabular}{|c|c|c|c|c|c|c|c|c|}
\hline \multirow[b]{3}{*}{ ADNI Feature } & \multirow{3}{*}{$\begin{array}{l}\text { Subject\# } \\
\text { Description }\end{array}$} & \multirow{2}{*}{\multicolumn{2}{|c|}{$\frac{\text { EMCI_C }}{49}$}} & \multirow{2}{*}{\multicolumn{2}{|c|}{$\begin{array}{c}\text { EMCI_NC } \\
335\end{array}$}} & \multirow[b]{3}{*}{$\begin{array}{l}\text { 6- } \\
\text { FT }\end{array}$} & \multirow[b]{3}{*}{$\begin{array}{l}9- \\
\text { FT }\end{array}$} & \multirow[b]{3}{*}{$\begin{array}{l}\text { 13- } \\
\text { FT }\end{array}$} \\
\hline & & & & & & & & \\
\hline & & Mean & SD & Mean & SD & & & \\
\hline DX & Diagnosis & - & - & - & - & - & - & - \\
\hline $\begin{array}{l}\text { Demographic information } \\
\text { PTRACCAT } \\
\text { AGE }\end{array}$ & $\begin{array}{l}\text { Patient Race } \\
\text { Patient Age }\end{array}$ & $\begin{array}{l}- \\
73.5\end{array}$ & $\begin{array}{l}- \\
6.47\end{array}$ & $\begin{array}{l}- \\
71.1\end{array}$ & $\begin{array}{l}- \\
7.49\end{array}$ & $\begin{array}{l}\checkmark \\
\checkmark\end{array}$ & $\begin{array}{l}\checkmark \\
\checkmark\end{array}$ & $\begin{array}{l}\checkmark \\
\checkmark\end{array}$ \\
\hline $\begin{array}{l}\text { Genetic Biomarkers } \\
\text { APOE4 }\end{array}$ & The number of e 4 alleles of APOE & .9 & .71 & .4 & .46 & - & $\checkmark$ & $\checkmark$ \\
\hline $\begin{array}{l}\text { Physical Biomarkers } \\
\text { Hippocampus } \\
\text { Ventricles }\end{array}$ & $\begin{array}{l}\text { Hippocampal volume } \\
\text { Ventricular volume }\end{array}$ & $\begin{array}{l}6875.2 \\
39282.7\end{array}$ & $\begin{array}{l}947.45 \\
21031.66\end{array}$ & $\begin{array}{l}7334.1 \\
34504.6\end{array}$ & $\begin{array}{l}910.20 \\
21394.49\end{array}$ & $\begin{array}{l}- \\
-\end{array}$ & $\begin{array}{l}\checkmark \\
\checkmark\end{array}$ & $\begin{array}{l}\checkmark \\
\checkmark\end{array}$ \\
\hline $\begin{array}{l}\text { Neuropsychological scales } \\
\text { ADAS13 } \\
\text { ADAS11 } \\
\text { FAQ } \\
\text { MMSE } \\
\text { RAVLT_immed } \\
\text { RAVLT_learn } \\
\text { RAVLT_forg } \\
\text { RAVLT_perc_forg }\end{array}$ & $\begin{array}{l}\text { 13-item AD Assessment Scale } \\
\text { 11-item AD Assessment Scale } \\
\text { Functional Activities Questionnaire } \\
\text { Mini-Mental State Examination } \\
\text { \# of words memorized over } 5 \text { trials } \\
\text { \# of words learned between trials 1-5 } \\
\text { \# of words forgotten between trials 5-6 } \\
\text { \% of words forgotten between trials 5-6 }\end{array}$ & $\begin{array}{l}15.8 \\
9.7 \\
4.1 \\
28.1 \\
34.5 \\
4.7 \\
5.1 \\
60.7\end{array}$ & $\begin{array}{l}6.02 \\
4.12 \\
4.38 \\
1.58 \\
8.39 \\
2.47 \\
2.54 \\
29.13\end{array}$ & $\begin{array}{l}13.3 \\
8.5 \\
1.82 \\
28.3 \\
40.3 \\
5.3 \\
4.1 \\
44.0\end{array}$ & $\begin{array}{l}5.41 \\
3.29 \\
2.50 \\
1.71 \\
11.40 \\
2.42 \\
2.64 \\
29.36\end{array}$ & $\begin{array}{l}\checkmark \\
\checkmark \\
\checkmark \\
\checkmark \\
- \\
- \\
- \\
-\end{array}$ & $\begin{array}{l}\checkmark \\
\checkmark \\
\checkmark \\
\checkmark \\
- \\
- \\
- \\
-\end{array}$ & $\begin{array}{l}\checkmark \\
\checkmark \\
\checkmark \\
\checkmark \\
\checkmark \\
\checkmark \\
\checkmark \\
\checkmark \\
\checkmark\end{array}$ \\
\hline
\end{tabular}

$E M C I_{-} C$ the converter group, $E M C I_{-} N C$ the stable group, $F T$ Feature Training

\section{Random forest classification model}

Random forests are an ensemble learning method for classification, regression, and other tasks that operate by constructing a multitude of decision trees at training time and outputting the class that is either the mode of the classes, in regards to a classifier, or the mean prediction of the individual trees for a regression model. Since random forests consist of a collection of decision trees that are trained with different data subsets and then averaged, this allows them to be tolerant of the problem of overfitting.

For our work (as seen in Figure 2), the random forest classifier has two potential classes eligible for its output, EMCI_C (patients that converted to AD) and EMCI_NC (patients that did not convert to $\mathrm{AD}$ ). These classes are voted on from each individual tree, which is then aggregated to provide an overall probability of $\mathrm{AD}$ conversion. Figure 3 shows an example of an individual tree. Random Forest classifiers also allow for individual input variable importance to be evaluated. As part of our work, we built an ad hoc prediction script that evaluates this variable importance at both the model and individual prediction levels. Initially, while training the model, this evaluation helped us determine which variables were most relevant for model inclusion. After the model has been trained, this variable importance ranking then helps to interpret the individual prediction results. 


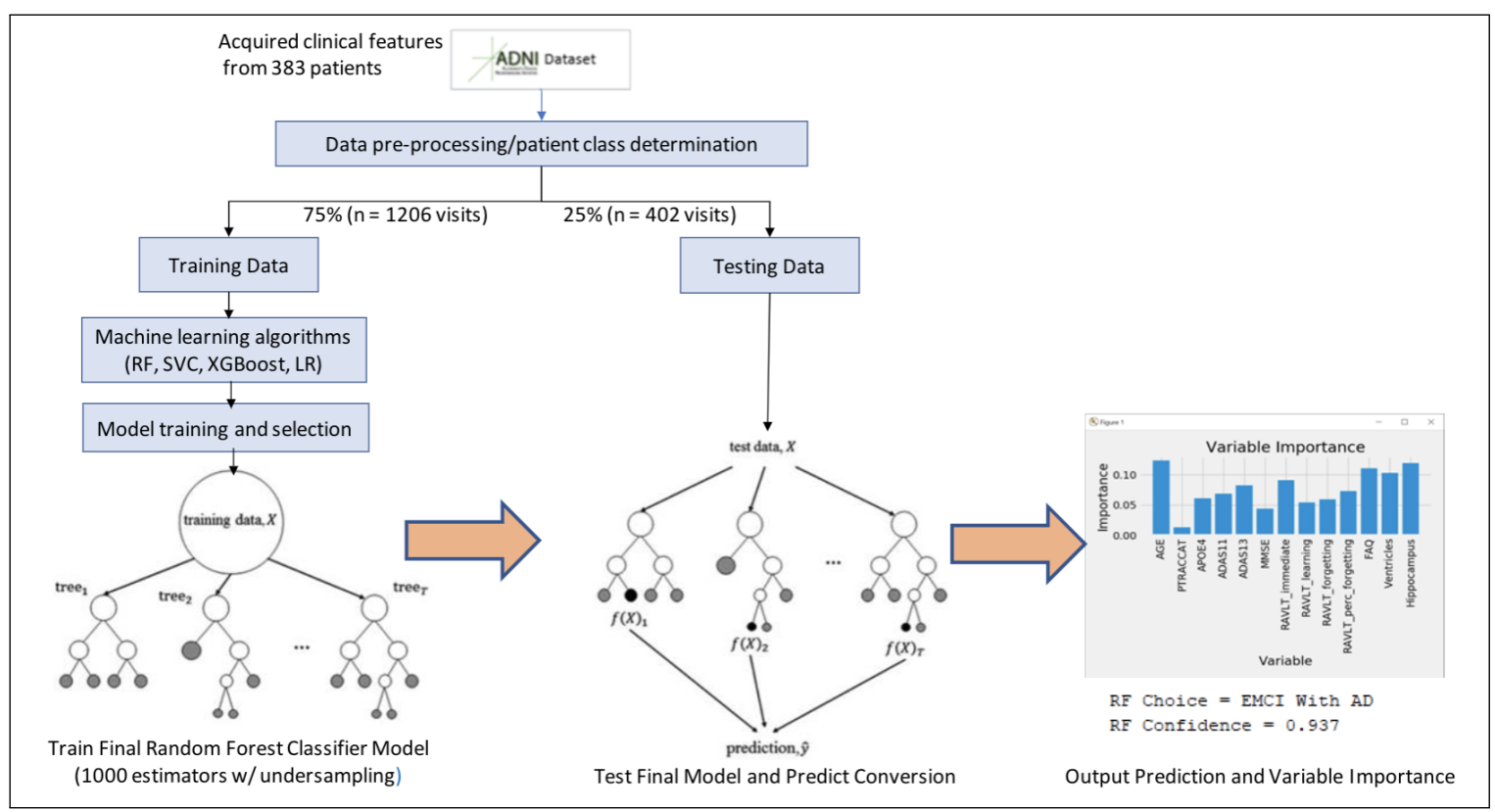

Fig 2. Model Workflow

\section{Balancing the data}

As a result of our imbalanced data set, where only $12.8 \%$ of the patients belonged to the minority class (EMCI_C); we perform a modified random forest algorithm that randomly over-samples each bootstrap sample. This provides a balance between the two classes so that the majority class is not always predicted, which would otherwise skew our accuracy results. This allows for the model to be trained against 2412 exam visits (1206 per class) rather than only the 1354 from the original data set train/test split. Table1 further demonstrates the evolution of the data set after over-sampling. We compared our over-sampling method against an under-sampling method that targeted the majority class and found a $3.1 \%$ increase in accuracy via the over-sampling process. This was also compared to class weight modifications, and over-sampling continued to be the best method for our work.

\section{Results}

\section{Demographic and clinical characteristics}

As can be seen across Figure 1 and Table 2, 383 EMCI patients were gathered from the ADNI database, of which 49 would convert to AD (EMCI_C), and 335 would not convert (EMCI_NC). The patients' average age was 71.4 , and $55.6 \%$ of the patients were men. There was a significant difference in age between the two groups $(\mathrm{P}<.05)$ according to the t-test.

Also shown in Table 2 are the genetic and physical biomarkers. The APOE4 and hippocampal volume differences were statistically significant between the EMCI_C and EMCI_NC groups, whereas the ventricular volume was not. For the neuropsychological scale scores, the ADAS13 and the FAQ features were significantly different $(\mathrm{P}<.05)$. The ADAS11 and the MMSE features were found not to be significantly different. The relationships between our features are further seen in Figure 5 as a Correlation Matrix. 
bioRxiv preprint doi: https://doi.org/10.1101/2020.12.17.423213; this version posted December 17, 2020. The copyright holder for this preprint (which was not certified by peer review) is the author/funder, who has granted bioRxiv a license to display the preprint in perpetuity. It is made available under aCC-BY 4.0 International license.

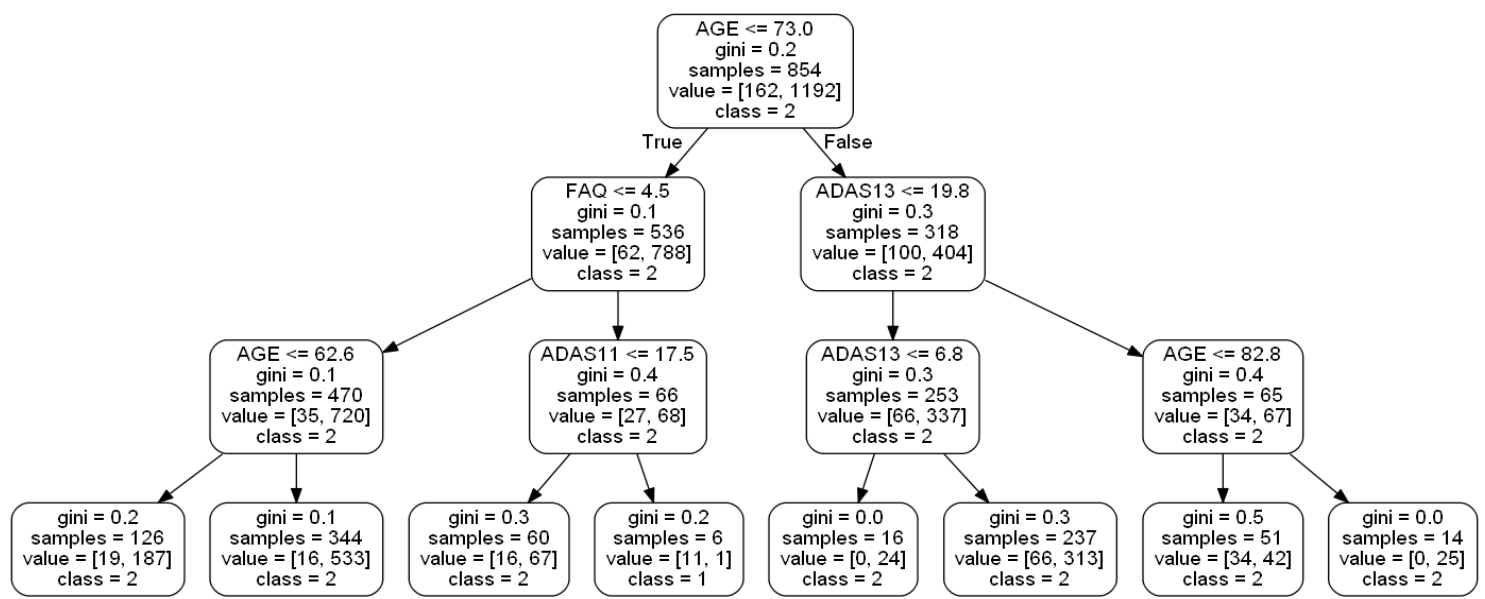

Fig 3. Example of a small random forest tree within our model

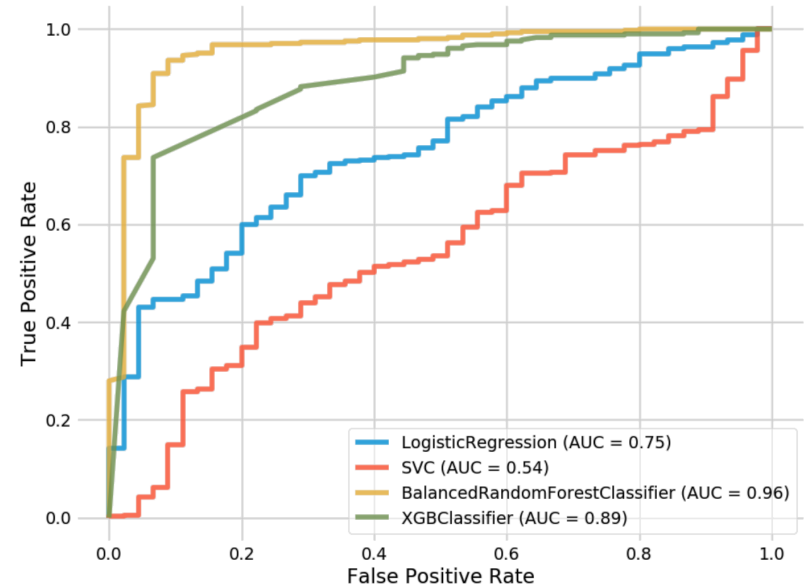

Fig 4. Receiver operating characteristic curves for Random Forest and comparison models.

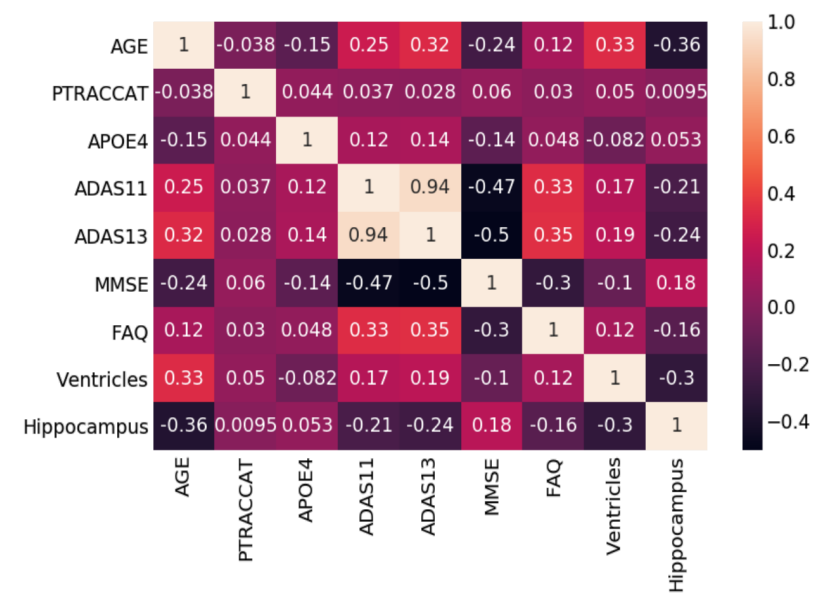

Fig 5. Random Forest Model Correlation Matrix 


\section{Model performance}

A workflow of our random forest model can be seen in Figure 2. This summarizes the training methodology as well as the prediction and variable importance output. After our pre-processing steps, we train a 1000 tree random forest model on 2412 exam visits against different feature groups to compare their results. While initially, using all 13 -features seemed to provide the highest accuracy at $91.6 \%$, we found that by removing the RAVLT features, our accuracy rose to $93.6 \%$ in the 9-feature group. We also tested a 6-feature group, which removed the RAVLT variables as well as the biomarker data; however, this saw the worst accuracy of the three groups at $89.2 \%$.

After running these feature group variations through our random forest training process, we decided to implement the same training data into a support vector classifier (SVC), an XGBoost classifier, and a Logistic Regression model for comparison. As these are commonly used for this problem, we considered this to be a reasonable comparative measure to the efficacy of our random forest model.

Support vector classifiers attempt to find the separating hyperplane that maximizes the distance of the closest points to the boundary of the class. These are typically effective in high dimensional spaces and have seen a fair amount of usage within the $\mathrm{AD}$ conversion prediction domain [12] [8]. In both the 9-feature and 13-feature groups, we found that our random forest model outperformed our SVC implementation (93.6\% vs. $90 \%$ and $91.6 \%$ vs. $90 \%$, respectively). The SVC did show higher accuracy than the 6 -feature RF model; however, the AUC was inferior on all SVC variations. The difference in AUC between our best RF variation (96\% AUC) and our best SVC variation ( $54 \%$ AUC) is shown in Figure 4. One observation when observing our SVC model is that it struggled to predict the negative class (conversion to AD) and predominantly chose the majority class. This was not the case with our balanced random forest model which was able to appropriately distinguish between both classes.

XGBoost is an implementation of gradient boosted decision trees that has seen success in structured data classification. While not being common in the AD conversion prediction space, we wanted to compare how our feature-selection would be handled by its algorithm. XGBoost resulted in the second-best overall method behind our top RF model and showed significantly better performance than the SVC and Logistic Regression implementations. For the 6 -feature group, XGBoost outperformed our RF model (89.8\% vs. $89.2 \%$ ). However, while performing better than SVC and Logistic Regression, the XGBoost model still saw less accuracy than the RF model at both the 9 and 13 feature groups, as seen in Table 3. When comparing AUC, one can see how well XGBoost performed (89\%) in relation to SVC (54\%) and Logistic Regression (75\%).

Finally, Logistic Regression was the last method that we leveraged for comparison. Logistic Regression calculates the probability of an event occurrence and can be used when the target variable is categorical. For this model, we observed a consistent .2\% increase in accuracy for each subsequently larger feature group. Additionally, while its AUC $(75 \%)$ underperformed in contrast to RF and XGBoost, it did significantly better than our best SVC model (54\%). Still, this did not result in a model that was close enough to warrant further consideration for our AD conversion problem.

As mentioned previously, our 9-feature random forest implementation with an accuracy of $93.6 \%$ and an AUC of $96 \%$ against a 383-patient data set represents our best model. While also using the ADNI data set, Grassi et al. [12] could achieve an AUC of $88 \%$ with an SVM that made predictions 3 years prior to AD onset. Huang et al. [8] also attained $80 \%$ accuracy and $84.6 \%$ AUC with an SVM model against the ADNI data set leveraging both clinical and MRI data looking 5 years prior to AD onset. As our approach differs by using Early Mild Cognitive Impairment patients (EMCI) rather than the broader MCI grouping used by other studies, we can predict conversion from 5-7 years prior to the onset of AD. Our outcome is state-of-the-art when 
Table 3. Performance of Random Forest vs Support Vector Classifier among various feature groups

\begin{tabular}{|l|l|l|l|l|l|l|}
\hline Feature Group & Accuracy & Precision & Recall & F1 Score & AUC & KS Statistic (p-value) \\
\hline Random Forest & & & & & & \\
6-Features & 0.892 & 0.907 & 0.980 & 0.942 & 0.88 & 0.91 \\
9-Features & $\mathbf{0 . 9 3 6}$ & $\mathbf{0 . 9 5 2}$ & 0.978 & $\mathbf{0 . 9 6 5}$ & $\mathbf{0 . 9 6}$ & $\mathbf{0 . 7 1}$ \\
13-Features & 0.916 & 0.916 & $\mathbf{0 . 9 9 8}$ & 0.955 & 0.93 & 0.82 \\
\hline Support Vector Classifier & & & & & & \\
6-Features & 0.900 & 0.900 & 1 & 0.948 & 0.52 & - \\
9-Features & 0.900 & 0.900 & 1 & 0.948 & 0.54 & - \\
13-Features & 0.900 & 0.900 & 1 & 0.948 & 0.55 & - \\
\hline Logistic Regression & & & & & & \\
6-Features & 0.894 & 0.902 & 0.990 & 0.944 & 0.76 & - \\
9-Features & 0.892 & 0.903 & 0.985 & 0.942 & 0.75 & - \\
13-Features & 0.896 & 0.904 & 0.990 & 0.945 & 0.75 & - \\
\hline XGBoost & & & & & & \\
6-Features & 0.898 & 0.904 & 0.993 & 0.946 & 0.87 & - \\
9-Features & 0.920 & 0.930 & 0.985 & 0.957 & 0.89 & - \\
13-Features & 0.907 & 0.921 & 0.980 & 0.950 & 0.88 & - \\
\hline
\end{tabular}

Table 4. State of the art MCI-to-AD Prediction

\begin{tabular}{l|l|l|l|l|l|l}
\hline Approach & Modalities & Data & Model & \multicolumn{2}{|l|}{ MCI-to-AD Prediction } & \multirow{2}{*}{ Year } \\
\cline { 5 - 6 } & & (subject size) & & ACC & AUC & \\
\hline Proposed Model (ours) & Clinical data & ADNI (383) & RF & $\mathbf{9 3 . 6 \%}$ & $\mathbf{9 6 \%}$ & 5 \\
Grassi et al. (2019) [12] & Clinical data & ADNI (550) & SVM & - & $88 \%$ & 3 \\
Huang et al. (2019) [8] & Clinical & ADNI (290) & SVM & $80 \%$ & $84.6 \%$ & 5 \\
& data/MRI & & & & & \\
Albright (2019) [11] & Clinical data & ADNI (1737) & MLP & - & $86.6 \%$ & 5 \\
Moore et al. (2019) [9] & Clinical data & ADNI (1627) & RF & $73 \%$ & $82 \%$ & 5 \\
Ghazi et al. (2019) [13] & MRI & ADNI (742) & RNN & - & $76 \%$ & 5 \\
\hline
\end{tabular}

comparing our accuracy and AUC to the previously published work for MCI-to-AD prediction as shown in Table 4.

\section{Assessment of model feature importance}

One advantage of using the random forest algorithm is that feature importance can be assessed at both the model and individual prediction levels. The model feature

importance of our three feature groupings can be seen in Figures 6-7 and Figure 9. As a random forest algorithm deals with different combinations of features in each of its' decision trees, this allows for the feature importance to be calculated based on how much the prediction error increases. This error is calculated per tree and is then averaged across the entire forest before being divided by the standard deviation [9].

For our 6-feature model, the three most important features are Age, FAQ, and ADAS13. For our top-performing 9-feature model, the top features are Age, Hippocampus, and Ventricles. Finally, for our 13-feature model, Age, Hippocampus, and FAQ score are the most important. The presence of hippocampal and ventricular volume towards the top explains why the absence of those features in our 6 -feature model resulted in diminished accuracy. By adding in the RAVLT features, our accuracy improved, but these were redundant with the other neuropsychological scale scores, so they were removed for our final model. Age was consistently seen as the best conversion predictor, which corresponds to the increased risk of AD at an older age [14]. Race (PTRACCAT) was routinely at the lowest feature importance between our models, but we did observe a decrease in accuracy upon its' removal. This is likely due to race 


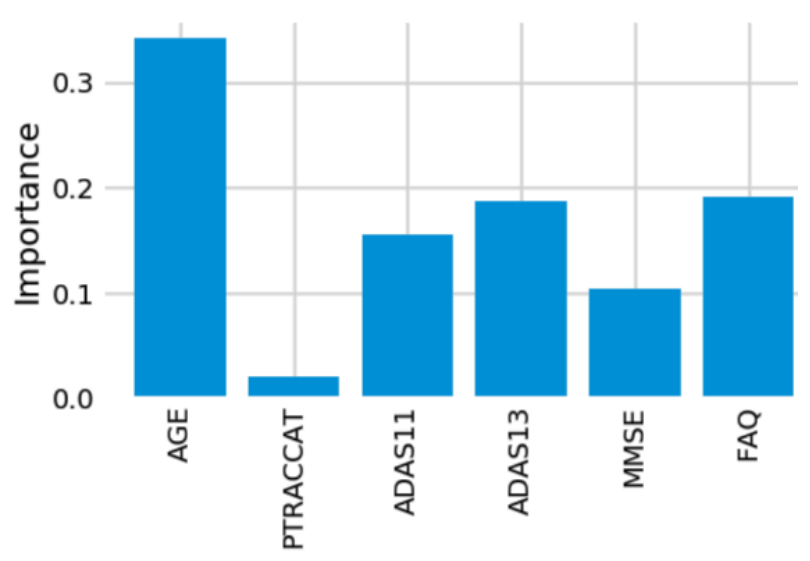

Fig 6. 6-Feature Model Feature Importance

having very little correlation with the other included features, whereas some neuropsychological scores exhibited signs of possible overlap (RAVLT).

For our best model, we also assess the permutation importance seen in Figure 8. This reduces the high cardinality bias seen in the feature importance charts by permuting against a held-out test set. This is done by each feature column being permuted against a baseline metric that was initially evaluated against the data set. The permutation importance is then established as the difference between the baseline metric and the feature column permutation. From this, we see that Age and FAQ maintain their high importance. However, APOE4 is now significantly more relevant in regards to the test set prediction.

Table 5 shows the average standard deviation differences from the mean out of the subjects that the model predicted incorrectly. We omit our PTRACCAT (Race) feature into this analysis, given that it is not a continuous variable. In total, 20 EMCI_C ground truth subjects and 9 EMCI_NC ground truth subjects were incorrectly classified. By analyzing the standard deviation differences, we can determine which feature was most abnormal compared to the average model prediction for that given class.

We do this by establishing the data set means and standard deviations per feature for both the EMCI_C and EMCI_NC classes. We then take each misclassified patient's feature values and subtract them by the corresponding mean, prior to dividing them by that feature's standard deviation value. This allows us to see which features were the most unusual at an individual patient level. Coupled with the feature importance ranking this gives us clearer insight into the model's prediction rationale. For example, the MMSE feature was 2.37 standard deviations away from its EMCI_C mean, which contributed to our model misclassifying those cases as EMCI_NC. Of the EMCI_C misclassifications, MMSE proved to be the most misleading feature. However, we still saw higher overall accuracy by including it within our model because of its relatively low feature importance.

Out of the EMCI_NC misclassifications, there was less clarity as to which feature was problematic. However, we do observe a standard deviation increase in our neuropsychological test scores across the nine misclassifications. This is indicative of the model believing these subjects' test scores to be similar to those of the EMCI_C class and thus making the false prediction. In future work, we will explore whether knowing these misleading instances can help our model's accuracy, but currently, it appears that these are outliers within the ADNI dataset. 
bioRxiv preprint doi: https://doi.org/10.1101/2020.12.17.423213; this version posted December 17, 2020. The copyright holder for this preprint (which was not certified by peer review) is the author/funder, who has granted bioRxiv a license to display the preprint in perpetuity. It is made available under aCC-BY 4.0 International license.

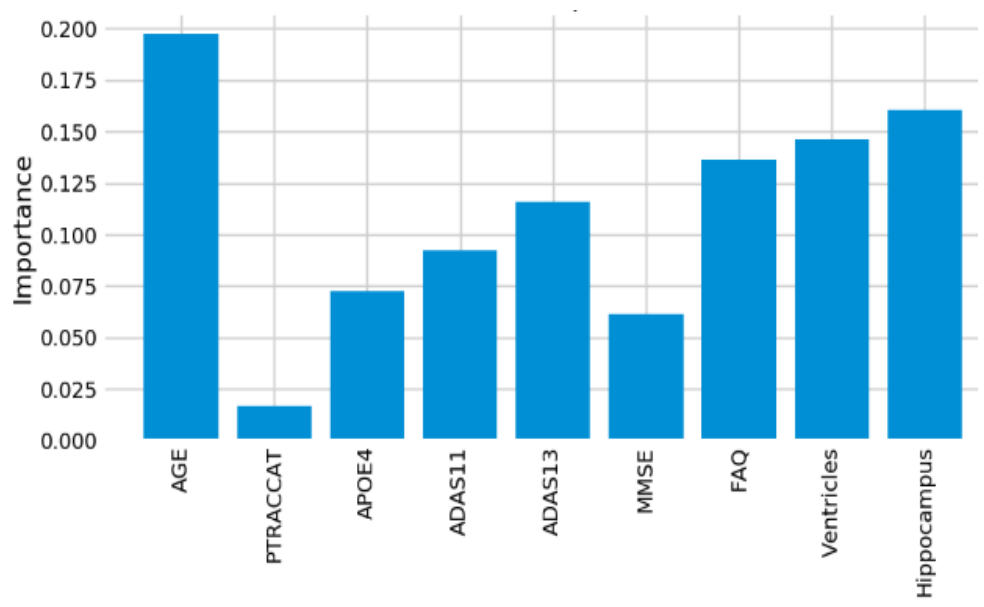

Fig 7. 9-Feature Model Feature Importance

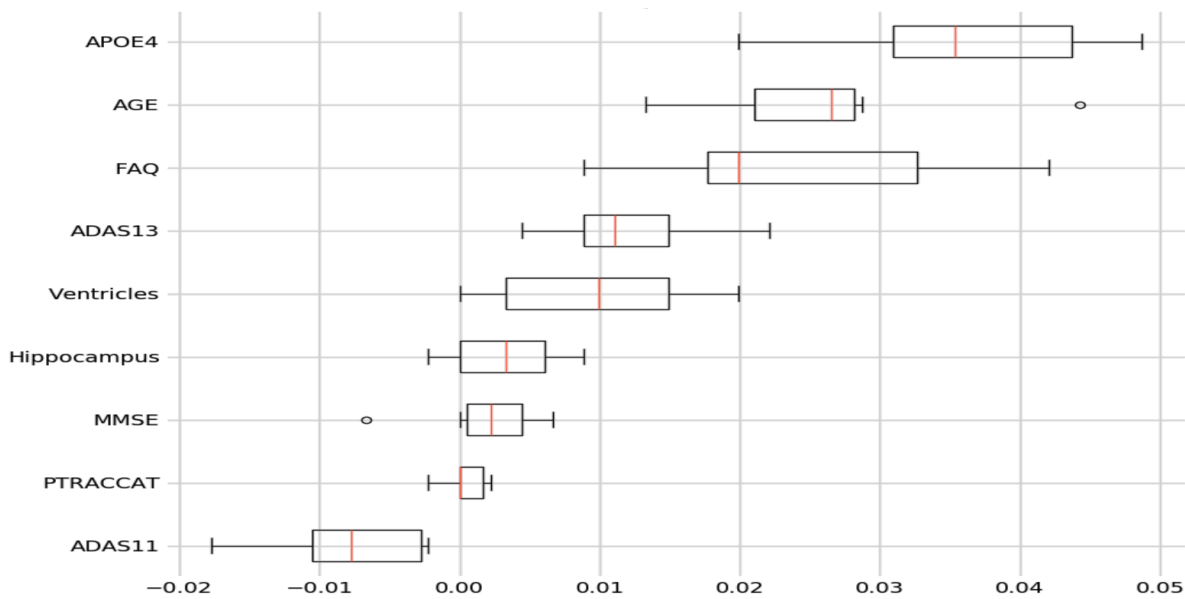

Fig 8. 9-Feature Model Permutation Importance

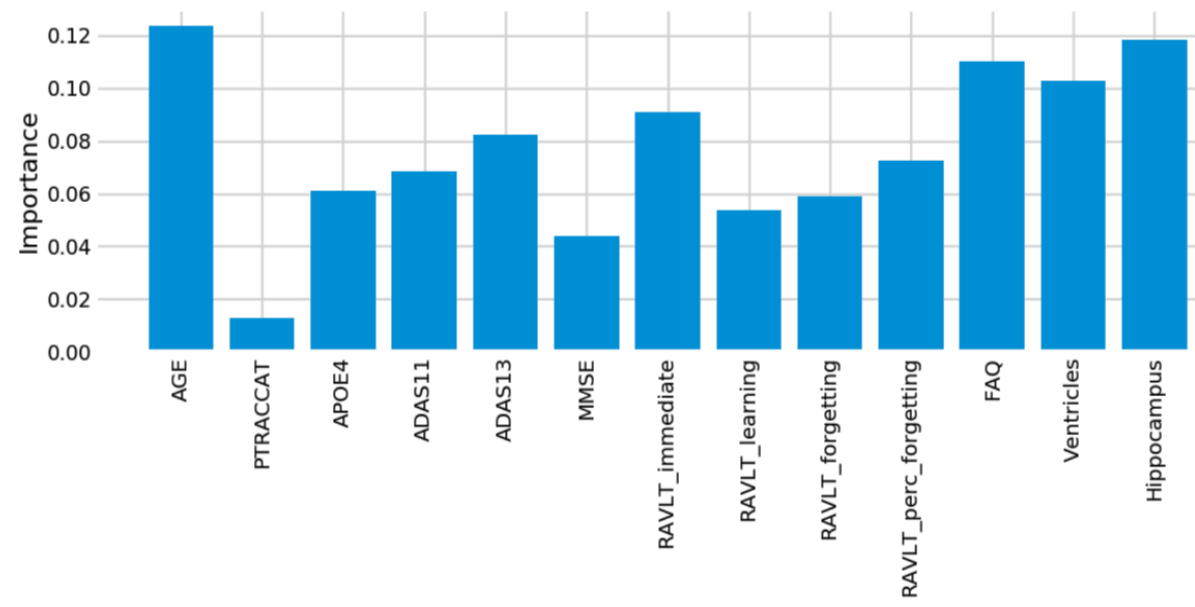

Fig 9. 13-Feature Model Feature Importance 
Table 5. Average standard deviation difference for incorrect predictions by ground truth class and feature

\begin{tabular}{|l|l|l|l|l|l|l|l|l|l|}
\hline & Record\# & Age & APOE4 & ADAS11 & ADAS13 & MMSE & FAQ & Ventricles & Hippocampus \\
\hline EMCI_C & 20 & 1.90 & 2.20 & 1.30 & 1.38 & $\mathbf{2 . 3 7}$ & 0.82 & 0.80 & 1.00 \\
\hline EMCI_NC & 9 & 1.74 & 2.26 & $\mathbf{2 . 8 7}$ & 2.46 & 2.68 & 2.61 & 1.13 & 2.76 \\
\hline
\end{tabular}

\section{Assessment of individual predictors' feature importance}

For the individual level, we can see which features specific to that patient made the largest difference in their prediction. An example of these prediction contributions can be seen in Figure 10 based on the test patient's features provided in Table 6. In this case, our model correctly predicted that this patient would convert to $\mathrm{AD}$ with an overall confidence of $90.4 \%$. This confidence is a reflection of the aggregate of all of the individual trees' votes within our forest.

From Figure 10 and the contributions listed in Table 6, we see that ventricular volume was the most essential feature for this patient as it contributed $24.2 \%$ towards the prediction. This was closely followed by their Functional Activities Questionnaire (FAQ) score which contributed $21.2 \%$ of the prediction. These differences in rank from the overall model importance graph stem from the patient's large deviations from the mean within those specific features. As this patient's ventricular volume was 24,285 , this is significantly less than the 34,505 mean ventricular volume for non-converting patients. Additionally, their FAQ score of 10 marks a notable difference from the 1.82 mean score of non-converting patients.

For this case, age did remain as one of the top 3 most essential features; however, it was not nearly as prominent for this individual, contributing only $11.8 \%$ towards the prediction. By performing additional individual predictions, we found that age consistently stays within the top 5 most important features, which explains its high importance once aggregated to the model importance level. Interestingly enough, hippocampal volume was not very important at all (2.5\% contribution) in this patient's prediction compared to the $16 \%$ contribution that it provided the overall model. This is due to the patient's hippocampal volume of 6,901 falling in-between the means of both the conversion $(6,875.2)$ and the non-conversion $(7,334.1)$ groups. Race (PTRACCAT) was the only feature that contributed to the wrong prediction, albeit only a $0.3 \%$ prediction contribution for this patient. Additional patients and their features' prediction contributions can be seen in Table 6 .

Table 7 shows the prediction contributions from five misclassified subjects. Similarly to Table 6 , the PC column represents the amount of each feature's contribution to the overall prediction. A positive value indicates the contribution towards the ground truth class, whereas a negative value denotes the contribution towards the incorrect class. As Table 5 is helpful for interpreting possibly misleading features at the model level, this individual PC metric allows us to better understand the model's decision making on a per subject basis.

\section{Discussion}

We have demonstrated that a random forest model can take clinical features and accurately predict MCI-to-AD conversion probability. Our RF classifier showed superior performance compared to competing SVM, XGBoost, and Logistic Regression implementations, including our own. It is also worth noting that the best models looked at all MCI patients, rather than the earlier EMCI subset. This gives our model the strength of predicting from 5-7 years prior to the onset of AD. Our results show that clinical features can also outperform MRI-based models. This is important as obtaining neuropsychological scores, a significant subset of our chosen features can be far more 


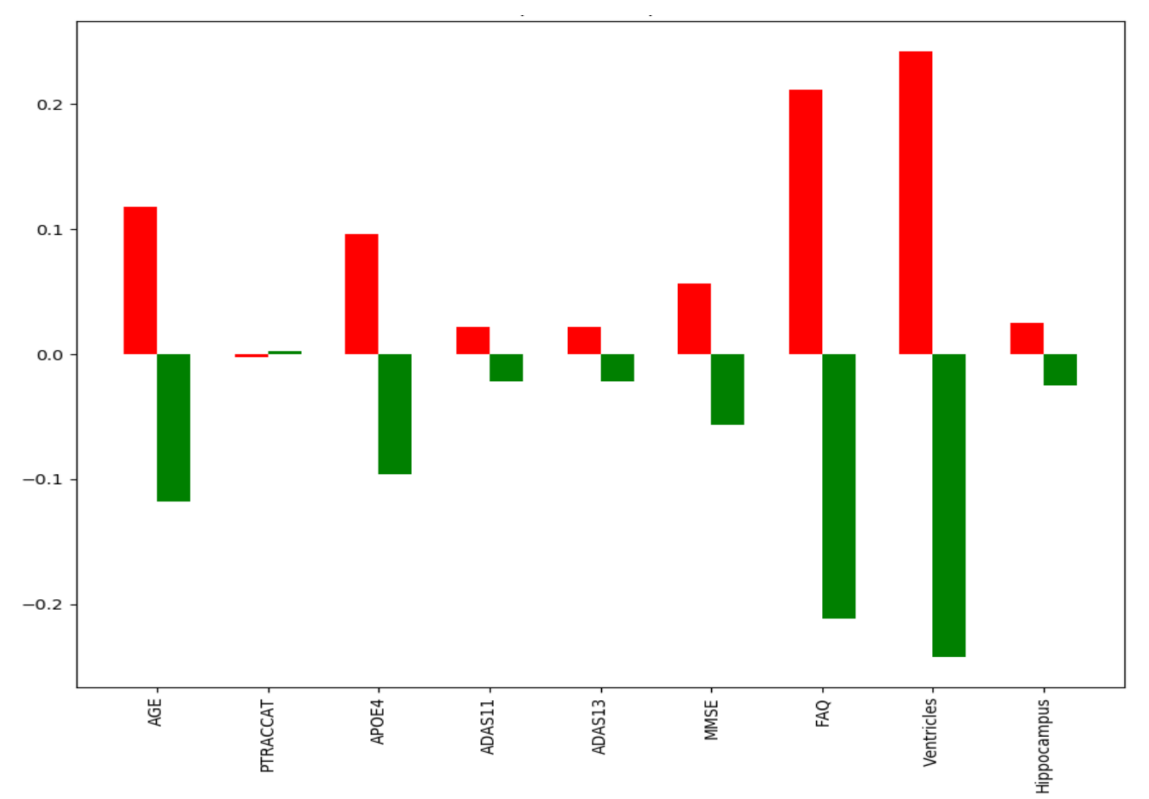

Fig 10. Feature Importance example for an individual patient

Table 6. Example features and prediction contributions (pc) from five correctly classified cases

\begin{tabular}{|l|l|l|l|l||l|l|l|l|l|l|}
\hline Subject & \multicolumn{2}{|c|}{ Subject 1 } & \multicolumn{2}{c||}{ Subject 2 } & \multicolumn{2}{c|}{ Subject 3 } & \multicolumn{2}{c|}{ Subject 4 } & \multicolumn{2}{c|}{ Subject 5 } \\
\hline Class & \multicolumn{2}{|c|}{ EMCI_C } & \multicolumn{2}{c|}{ EMCI_C } & \multicolumn{2}{c|}{ EMCI_NC } & \multicolumn{2}{c|}{ EMCINC } & \multicolumn{2}{c|}{ EMCI_NC } \\
& Value & PC & Value & PC & Value & PC & Value & PC & Value & PC \\
\hline Age & 68 & 0.11 & 77 & 0.118 & 59 & 0.047 & 64 & 0.024 & 81 & 0.001 \\
PTRACCAT & 7 & -0.001 & 7 & -0.003 & 7 & 0.001 & 7 & 0 & 7 & 0.008 \\
APOE4 & 2 & 0.248 & 1 & 0.096 & 0 & 0.045 & 0 & 0.018 & 1 & -0.002 \\
ADAS11 & 13 & 0.039 & 9 & 0.022 & 13 & -0.002 & 2 & 0.01 & 14 & 0.009 \\
ADAS13 & 20 & 0.042 & 17 & 0.022 & 16 & -0.001 & 4 & 0.024 & 21 & -0.005 \\
MMSE & 29 & 0.006 & 26 & 0.057 & 29 & 0.015 & 28 & 0.001 & 27 & -0.008 \\
FAQ & 0 & -0.036 & 10 & 0.212 & 4 & -0.037 & 1 & 0.025 & 1 & 0.056 \\
Ventricles & 38627 & 0.145 & 24285 & 0.242 & 22275 & -0.03 & 11776 & -0.001 & 30260 & -0.001 \\
Hippocampus & 7853 & 0.173 & 6901 & 0.025 & 8303 & 0.023 & 7960 & 0.013 & 6288 & 0.015 \\
\hline
\end{tabular}

affordable and less intensive than obtaining a patient's MRI imaging. With a more flexible approach, the expectation is that this predictor would be easier to deploy into a clinical setting.

In our experiments with the feature groupings, we found neuropsychological scores to be the most reliable and essential feature subset as we always experienced lower model accuracy with their exclusion. Performing tests on individual predictors also showed their weaknesses as each predictor demonstrated improved accuracy when coupled with an additional predictor. Even the neuropsychological scores by themselves exhibited signs of subjectivity, which were remediated by including biomarker and demographic features.

From our individual case analysis we see how important the FAQ and APOE4 features can be, as well as how misleading MMSE can be in some instances. This is interesting in that it shows while MMSE does increase model performance, there is some overlap between the two classes when dealing with that feature's outliers. For future work it will be important to determine if those outlying features can be identified and mitigated to then improve model performance. By combining the average standard deviation difference findings along with the prediction contribution results, we can 
Table 7. Example features and prediction contributions (pc) from five misclassified cases

\begin{tabular}{|l|l|l|l|l||l|l|l|l|l|l|}
\hline Subject & \multicolumn{2}{|c|}{ Subject 1 } & \multicolumn{2}{c||}{ Subject 2 } & \multicolumn{2}{c|}{ Subject 3 } & \multicolumn{2}{c|}{ Subject 4 } & \multicolumn{2}{c|}{ Subject 5 } \\
\hline \multirow{2}{*}{ Ground Truth } & \multicolumn{2}{|c|}{ EMCI_C } & \multicolumn{2}{c|}{ EMCI_C } & \multicolumn{2}{c|}{ EMCI_C } & \multicolumn{2}{c|}{ EMCI_NC } & \multicolumn{2}{c|}{ EMCI_NC } \\
\cline { 2 - 10 } & Value & PC & Value & PC & Value & PC & Value & PC & Value & PC \\
\hline Age & 69.1 & 0.008 & 73 & 0.337 & 75.9 & 0.014 & 79.8 & 0.106 & 63.6 & 0.009 \\
PTRACCAT & 7 & 0.003 & 7 & -0 & 7 & -0.003 & 7 & -0.006 & 7 & -0.002 \\
APOE4 & 2 & 0.138 & 0 & -0.022 & 1 & 0.017 & 0 & -0.041 & 0 & -0.037 \\
ADAS11 & 5 & -0.006 & 4 & 0.013 & 10 & -0.017 & 12 & 0.019 & 27 & 0.208 \\
ADAS13 & 10 & -0.008 & 9 & 0.025 & 16 & 0.024 & 17 & -0.013 & 37 & 0.346 \\
MMSE & 26 & 0.023 & 29 & -0.011 & 30 & -0.013 & 30 & -0.002 & 19 & -0.025 \\
FAQ & 4 & 0.007 & 3 & 0.039 & 2 & -0.021 & 5 & 0.099 & 17 & 0.158 \\
Ventricles & 35280.12 & -0.006 & 32379 & -0.031 & 62746 & 0.034 & 69583 & -0.043 & 35280.12 & -0.019 \\
Hippocampus & 5576 & 0.158 & 7835 & -0.02 & 6749 & 0 & 5437 & 0.256 & 7223.86 & -0.021 \\
\hline
\end{tabular}

confidently interpret the model's behavior and provide an efficient methodology for feature selection.

Additionally, our methods for oversampling an initially imbalanced data set can be of use throughout the medical research domain. With many medical data sets consisting of similar target class imbalance, our process enhances bagging algorithms by

augmenting more samples for the minority classes. For our purpose, this was only tested within a binary classification problem, however we will be extending this technique to multi-class problems.

One limitation of this study is that all of the patients were from the ADNI data set. While our accuracy was verified by splitting our data across multiple instances, we did not test the population outside of the ADNI participants. The inclusion of other data sets into our model would help account for even more significant variations and will be a target for future work.

In the future, we would like to combine this clinical features dependent model with our prior diffusion tensor imaging model [15] in order to create an ensemble predictor that can handle a large variety of available patient information. This would allow for greater flexibility for patient input data while maintaining high accuracy in the prediction. Additionally, it is currently difficult to differentiate between the sub-types of dementia when a patient presents with cognitive and memory decline [16]. This can lead to an inaccurate treatment plan if the patient is misdiagnosed. Having the ability to predict additional sub-types at such an early stage would help significantly with pharmacological management [17]. Researching the differences between these sub-types based on this study's clinical features will be a subject of our future work.

In summary, we created a balanced random forest model based on multiple features to predict the MCI-to-AD conversion probability. In addition, we determined which features were most important for the overall model, as well as for individual patient predictions. We also took advantage of oversampling methods to better balance the target classes. As early detection is critical for both clinical trial enrollment and cost-effective treatment plans, we expect our work to help in clinical diagnosis as well as establishing treatment timelines. Our random forest model achieved state-of-the-art performance with an accuracy of $93.6 \%$ and showed that the combination of demographic, neuropsychological scores and biomarker features could be used to predict which EMCI patients are at a higher risk of AD.

\section{Acknowledgement}

Data collection and sharing for this project was funded by the Alzheimer's Disease Neuroimaging Initiative (ADNI) (National Institutes of Health Grant U01 AG024904) 
and DOD ADNI (Department of Defense award number W81XWH-12-2-0012). ADNI is funded by the National Institute on Aging, the National Institute of Biomedical Imaging and Bioengineering, and through generous contributions from the following: AbbVie, Alzheimer's Association; Alzheimer's Drug Discovery Foundation; Araclon Biotech; BioClinica, Inc.; Biogen; Bristol-Myers Squibb Company; CereSpir, Inc.; Cogstate; Eisai Inc.; Elan Pharmaceuticals, Inc.; Eli Lilly and Company; Eurolmmun; F. Hoffmann-La Roche Ltd and its affiliated company Genentech, Inc.; Fujirebio; GE Healthcare; IXICO Ltd.; Janssen Alzheimer Immunotherapy Research \& Development LLC.; Lumosity; Lundbeck; Merck \& Co., Inc.; Meso Scale Diagnostics, LLC.; NeuroRx Research; Neurotrack Technologies; Novartis Pharmaceuticals Corporation; Pfizer Inc.; Piramal Imaging; Servier; Takeda Pharmaceutical Company; and Transition Therapeutics. The Canadian Institutes of Health Research is providing funds to support ADNI clinical sites in Canada. Private sector contributions are facilitated by the Foundation for the National Institutes of Health [18]. The grantee organization is the Northern California Institute for Research and Education, and the study is coordinated by the Alzheimer's Therapeutic Research Institute at the University of Southern California. ADNI data are disseminated by the Laboratory for Neuro Imaging at the University of Southern California.

\section{References}

1. International D. World Alzheimer Report 2011: The benefits of early diagnosis and intervention; World Alzheimer Report 2011: The benefits of early diagnosis and intervention; 2011. Available from: www.alz.co.uk/worldreport2011.

2. Random Forests Definition - DeepAI;. Available from: https: //deepai.org/machine-learning-glossary-and-terms/random-forest.

3. Sarica A, Cerasa A, Quattrone A. Random Forest Algorithm for the Classification of Neuroimaging Data in Alzheimer's Disease: A Systematic Review. Frontiers in Aging Neuroscience. 2017;9(OCT):329. doi:10.3389/fnagi.2017.00329.

4. Weiner MW, Veitch DP, Aisen PS, Beckett LA, Cairns NJ, Green RC, et al.. The Alzheimer's Disease Neuroimaging Initiative: A review of papers published since its inception; 2013.

5. Ganz M, Greve DN, Fischl B, Konukoglu E. Relevant feature set estimation with a knock-out strategy and random forests. NeuroImage. 2015;122:131-148. doi:10.1016/j.neuroimage.2015.08.006.

6. Lebedev AV, Westman E, Van Westen GJP, Kramberger MG, Lundervold A, Aarsland D, et al. Random Forest ensembles for detection and prediction of Alzheimer's disease with a good between-cohort robustness. NeuroImage: Clinical. 2014;6:115-125. doi:10.1016/j.nicl.2014.08.023.

7. Li H, Liu Y, Gong P, Zhang C, Ye J. Hierarchical interactions model for predicting Mild Cognitive Impairment (MCI) to Alzheimer's Disease (AD) conversion. PLoS ONE. 2014;9(1). doi:10.1371/journal.pone.0082450.

8. Huang K, Lin Y, Yang L, Wang Y, Cai S, Pang L, et al. ARTICLE OPEN A multipredictor model to predict the conversion of mild cognitive impairment to Alzheimer's disease by using a predictive nomogram. Neuropsychopharmacology;doi:10.1038/s41386-019-0551-0. 
9. Moore PJ, Lyons TJ, Gallacher J. Random forest prediction of Alzheimer's disease using pairwise selection from time series data. PLOS ONE. 2019;14(2):e0211558. doi:10.1371/journal.pone.0211558.

10. ADNI — Study Design;. Available from: http://adni.loni.usc.edu/study-design/.

11. Albright J. Forecasting the progression of Alzheimer's disease using neural networks and a novel preprocessing algorithm. Alzheimer's and Dementia: Translational Research and Clinical Interventions. 2019;5:483-491. doi:10.1016/j.trci.2019.07.001.

12. Grassi M, Rouleaux N, Caldirola D, Loewenstein D, Schruers K, Perna G, et al. A novel ensemble-based machine learning algorithm to predict the conversion from mild cognitive impairment to Alzheimer's disease using socio-demographic characteristics, clinical information, and neuropsychological measures. Frontiers in Neurology. 2019;10(JUL). doi:10.3389/fneur.2019.00756.

13. Ghazi MM, Nielsen M, Pai A, Cardoso MJ, Modat M, Ourselin S, et al. Robust training of recurrent neural networks to handle missing data for disease progression modeling; 2018.

14. Guerreiro R, Bras J. The age factor in Alzheimer's disease. Genome Medicine. 2015;7(1):1-3. doi:10.1186/s13073-015-0232-5.

15. Velazquez M, Anantharaman R, Velazquez S, Lee Y. RNN-Based Alzheimer's Disease Prediction from Prodromal Stage using Diffusion Tensor Imaging. 2019 IEEE International Conference on Bioinformatics and Biomedicine (BIBM) RNN-Based. 2019;(Mci):1665-1672. doi:10.1109/bibm47256.2019.8983391.

16. Elahi FM, Miller BL. A clinicopathological approach to the diagnosis of dementia; 2017. Available from: /pmc/articles/PMC5771416/?report=abstracthttps: //www.ncbi.nlm.nih.gov/pmc/articles/PMC5771416/.

17. Iddi S, Li D, Aisen PS, Rafii MS, Thompson WK, Donohue MC. Predicting the course of Alzheimer's progression. Brain Informatics. 2019;6(1). doi:10.1186/s40708-019-0099-0.

18. Home - FNIH;. Available from: https://fnih.org/. 